\title{
The Effect of Specific Sling Exercises on the Functional Movement Screen Score in Adolescent Volleyball Players: A Preliminary Study
}

\author{
by \\ Paweł Linek ${ }^{1}$ Edward Saulicz'1, Andrzej Myśliwiec ${ }^{1}$, Monika Wójtowicz², \\ Tomasz Wolny ${ }^{1}$
}

\begin{abstract}
The existing data indicate that the result of the Functional Movement Screen (FMS) test influences the likelihood of subsequent injury in professional athletes. Therefore, exercises increasing test scores of the FMS may be useful at various stages of sports activity. This study evaluated the effects of the NEURAC sling exercises method on the FMS test score in teenage volleyball players. The study was conducted on 15 volleyball players aged 14 years. The FMS test was performed three times interspersed with a two-month interval. Between the first and the second assessment, neither additional treatment nor training was applied, while between the second and the third assessment, the participants performed stabilisation exercises based on the NEURAC method. Training was carried out twice a week, for eight weeks. The analysis showed that between the first and the second measurement, no significant differences occurred. The use of specific sling exercises caused a significant improvement in FMS results $(p \leq 0.01)$ between the first and the third, as well as the second and the third measurement. The applied stabilisation exercises based on the NEURAC method positively influenced the FMS test result in male subjects practicing volleyball. Performance of such exercises also resulted in more than $90 \%$ of the subjects having a total FMS test score $\geq 17$, which may be important in the prevention of injuries. The preliminary results indicate that this type of exercise should be included in a teenage volleyball training routine.
\end{abstract}

Key words: stability exercise; Functional Movement Screen; injury; prevention.

\section{Introduction}

The Functional Movement Screen (FMS) is a battery of motor tests, wherein the proper execution of each of the tests requires the ability to perform a movement in three dimensions. The FMS evaluates the quality of the basic locomotor pattern and any possible limitations or asymmetry in the movement patterns performed. These tests are also used to assess pain in athletes, muscle strength, joint stability in the lower limbs, muscle flexibility, balance and proprioception
(Narducci et al., 2011). The FMS is considered a comprehensive tool evaluating motor control through functional movement and dynamic coordination analysis (Cook et al., 2006a, b).

The FMS has also been used as an injury prediction tool in competitive athletes. Recent studies have shown that athletes with an FMS score under 14 were more likely to sustain an injury than their peers who received more than 14 points (Kiesel et al., 2007, 2011; Chorba et al.,

\footnotetext{
1 - Department of Kinesitherapy and Special Methods in Physiotherapy, The Jerzy Kukuczka Academy of Physical Education, Katowice, Poland.

2- The Walerian Eukasiński General High School nr 7 in Dąbrowa Górnicza, Poland.
} 
2010). Another study has shown that, regardless of the score obtained in the FMS test, asymmetry in movements pointed to a more than 2-fold higher risk of injury (Kiesel et al., 2008). Many authors have indicated that in addition to improper motor control or weakness of deep muscles, one of the factors inducing the formation of the injury may be muscle imbalance (Chorba et al., 2010; Kiesel et al., 2011). Longitudinal studies clearly show that in professional female athletes with improper motor control of the body, knee injuries are more common (Zazulak et al., 2007a, b).

In order to prevent injuries and enhance performance in athletes, rehabilitation and core stability exercises are used. Stanton et al. (2004) argue that core stability exercises focus on muscles in an equal manner using body mass or relatively light loads while lying down, seated or standing on unstable equipment. One of the methods used in therapy is the NEURAC sling exercise (formerly SET), which is expected to improve trunk muscle activation based on the performance of active exercises and with the aid of sling exercise equipment. This therapy aims at muscle relaxation, increasing range of motion and traction and stabilising musculature as well as sensorimotor exercises. NEURAC is also based on the neuromuscular activation principle via highstrength static and dynamic contraction exercises (Kirkesola, 2001, 2009).

Regardless of the sports level, prevention of injuries is one of the most important goals in sports medicine and physical therapy. This issue is of great importance at the professional level, where injury can prevent proper preparation, or completely excludes the possibility of participating in competitions. At the amateur level, often in youth sports clubs, the injury prevention aspect is not as highly stressed. However, comprehensive motor development at a younger age can bring significant benefits in the future. Given that the scores for the FMS test have a predictive value with regard to later susceptibility to injuries in competitive athletes (Kiesel et al., 2007, 2011; Chorba et al., 2010), the aim of the present study was to evaluate how exercises based on the NEURAC method would affect the FMS test scores in teenage volleyball players. Our hypothesis was that the NEURAC exercises would lead to significant improvements in the FMS test results.

\section{Material and Methods}

\section{Experimental Approach}

Teenage volleyball players were invited to participate in the research. To reduce possible confounding factors, the subjects were monitored for several months. Triple FMS test results (with all components) constituted the dependent variables, while the observation periods between assessment 1 and 2, 1 and 3, and 2 and 3 constituted the independent variables. Between assessment 2 and 3, the experimental group followed an eight week training program including stabilisation exercises based on the NEURAC method.

Subjects

The study was conducted on a randomly selected group of young male volleyball players ( $\mathrm{N}=17$; age, 14 years; body height, $173.1 \pm 8.65$ $\mathrm{cm}$; body mass, $59.1 \pm 9.41 \mathrm{~kg}$; BMI, $19.6 \pm 2.29$ $\mathrm{kg} / \mathrm{m}^{2}$ ). All participants trained for approximately $4.5 \mathrm{~h}$ per week and had at least 4 year experience practicing volleyball prior to the study.

The subjects were informed about the purpose and conduct of the study, and agreed to participate in the research (consent was required from the participant and a parent). The entire procedure was carried out according to the Declaration of Helsinki.

\section{Procedures}

\section{Examination}

The subjects completed the FMS test three times. The first two assessments had been conducted prior to stabilization training was applied (the interval between the tests was two months), and the third assessment was performed after completion of the eight week training program based on the NEURAC method (Figure 1). Only 15 subjects completed the third assessment, because two athletes had withdrawn from the study due to injury or personal matters. All measurements were conducted by the same qualified and experienced person who was not informed of the purpose of the research.

All three assessments consisted of testing conducted in the same facility, after a warm-up lasting 15 minutes. The examination of athletes was carried out in strictly predetermined order, i.e. the FMS tests were performed at the beginning of a training session, prior to technical and tactical 
exercises. The applied FMS tests consisted of seven motor tasks: a deep squat, a hurdle step, an in-line lunge, a shoulder mobility test, an active straight leg raise, a rotary stability test and a trunk stability push-up. All movement tasks were assessed on a scale from 0 to 3 , where 3 meant the proper execution of the locomotor pattern; 2 meant the execution of the locomotor pattern with elements of compensation; 1 meant the inability to perform the motor pattern; 0 indicating pain during movement. Each trial was performed twice, and the better of the two results was registered for further analysis (Cook et al., 2006a, b). The maximum number of points amounted to 21. Regarding tests carried out on both sides of the body, the overall FMS test result taken into account was solely the result of the weaker side.

Stabilisation training

Stabilisation training was conducted in the gym, following a $15 \mathrm{~min}$ warm-up. The exercises were performed in strictly predetermined order, in closed kinematic chains using slings attached to the gym horizontal bar and ladders. The exercises were carried out twice a week, for eight weeks. Each exercise was performed in two sets of eight repetitions. In each repetition, the subjects had to keep the final position for three seconds. Between sets of exercises a five-minute rest period was allowed. The stabilization exercises, which lasted about 20 min, were conducted on a normal training day, always with a prior warm-up and before the subsequent volleyball workout. The training protocol was completed by only 15 subjects, 14 of them had $100 \%$ attendance, and one player did not participate in three training sessions due to illness.

All exercises were designed so that they could also be applied during normal volleyball training. The order and description of exercises were as follows:

1. Blocking exercises:

A) Starting position (SP): The player is lying face down, the upper limbs bent at the elbows, hands placed on slings, the body is in one line. Movement: lifting the torso up (in line) with straightening the elbows. At the beginning, the subjects supported themselves on the knees and in the next training session, on the toes (Figures $2 b$ and $c$ ).

B) SP: as described above. Movement: moving the upper limbs forward. As a modification, a sensorimotor pillow was introduced to the exercise and put under the toes (Figure 2d).

C) SP: The player is lying back, the upper limbs bent at the elbows, hands on slings, considering lower limbs, only the heels are in contact with the ground. Movement: lifting the torso up, straightening the elbows and lifting the upper limbs (Figures $3 a$ and $b$ ).

2. Attack exercises:

A) SP: The player is face down, hands on slings, the body in a straight line, supported on the toes. Movement: the player raises up the trunk, raising a hand forward (Figure 2a).

B) SP: The player is kneeling straight (knees on a sensorimotor pillow), hands on slings, arms in internal rotation. Movement: sweeping movement by attacking the upper limb (Figure 3c).

\section{Statistical analysis}

In order to compare the differences between the results of all FMS tests, Friedmen's analysis was used (a nonparametric test for repeated measurements). For the significant main effect, post-hoc ANOVA Friedman's analysis was performed. Statistically significant differences were assumed at $p<0.05$.

\section{Results}

The results presented in Tables 1 and 2 indicate the differences between the following assessments considering the results of a deep squat, a trunk stability push-up, a rotator stability test on both sides of the body and the total value of the FMS. Post-hoc ANOVA Friedmen's analysis showed that significant differences were observed only between the first and the third measurement, as well as the second and the third measurement $(p \leq 0.01)$. No differences were found between measurements 1 and 2. Based on the average (Table 1) and actual rank (Table 2), there was a significant improvement of points obtained for the FMS tests, after the application of stabilisation training $(p \leq 0.01)$.

A significant number of the subjects scored the maximum points in the first measurement $-\mathrm{a}$ hurdle step on both sides of the body and shoulder mobility testing on the right side. Each time, 13 to 15 subjects performed an active straight leg raise on the left side (12/15) and the 
right side, and shoulder mobility assessment on the left side (11/15), thus, it was impossible to evaluate the real impact of stabilisation training on these elements of the FMS test (Table 2).

\begin{tabular}{|c|c|c|c|c|}
\hline \multirow{2}{*}{$\begin{array}{c}\text { First } \\
\text { measurement }\end{array}$} & Normal & \multirow{2}{*}{$\begin{array}{c}\text { Second } \\
\text { measurement }\end{array}$} & Normal + stability & \multirow{2}{*}{$\begin{array}{c}\text { Third } \\
\text { measurement }\end{array}$} \\
\hline & training & & training & \\
\hline
\end{tabular}

Figure 1

\section{Stages of the experiment}

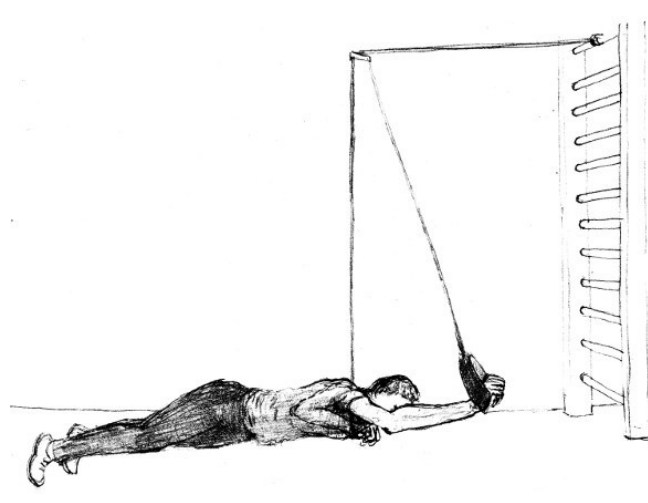

a

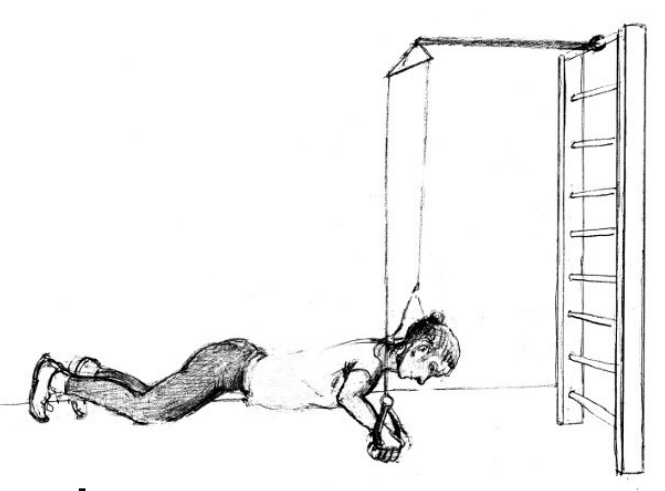

b

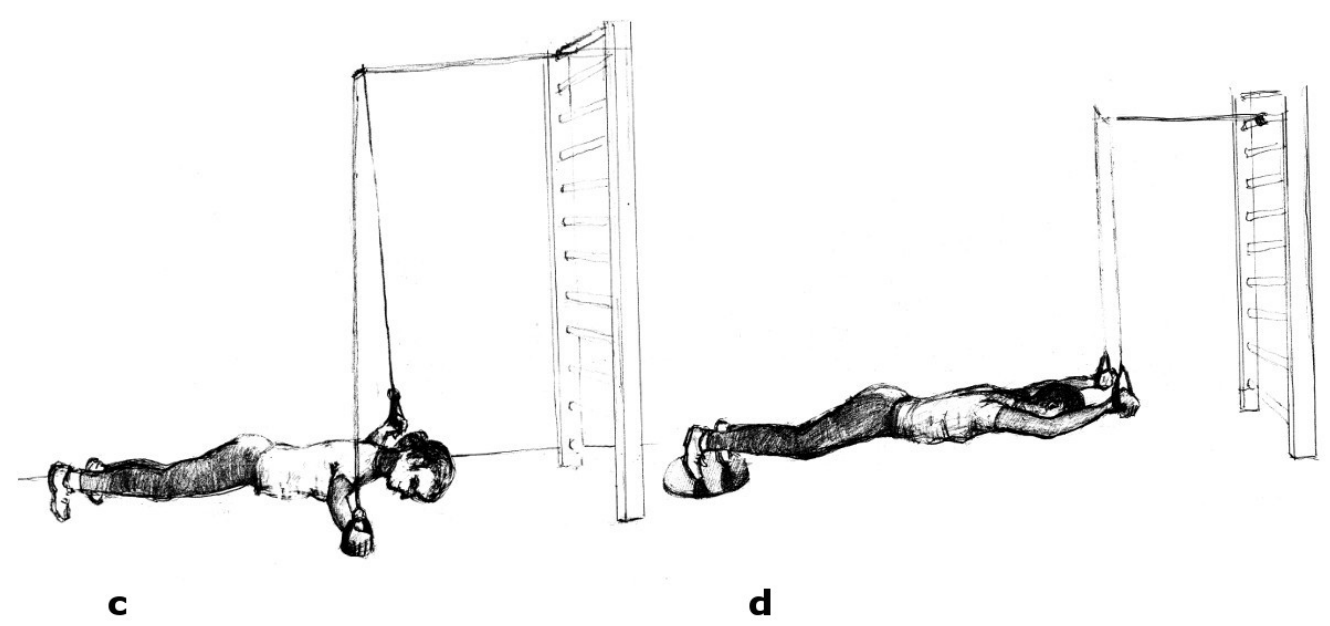

Figure 2

Selected exercises in posterior tilting of the body (attack exercise - $a$; blocking exercises $-b, c, d$ ). 


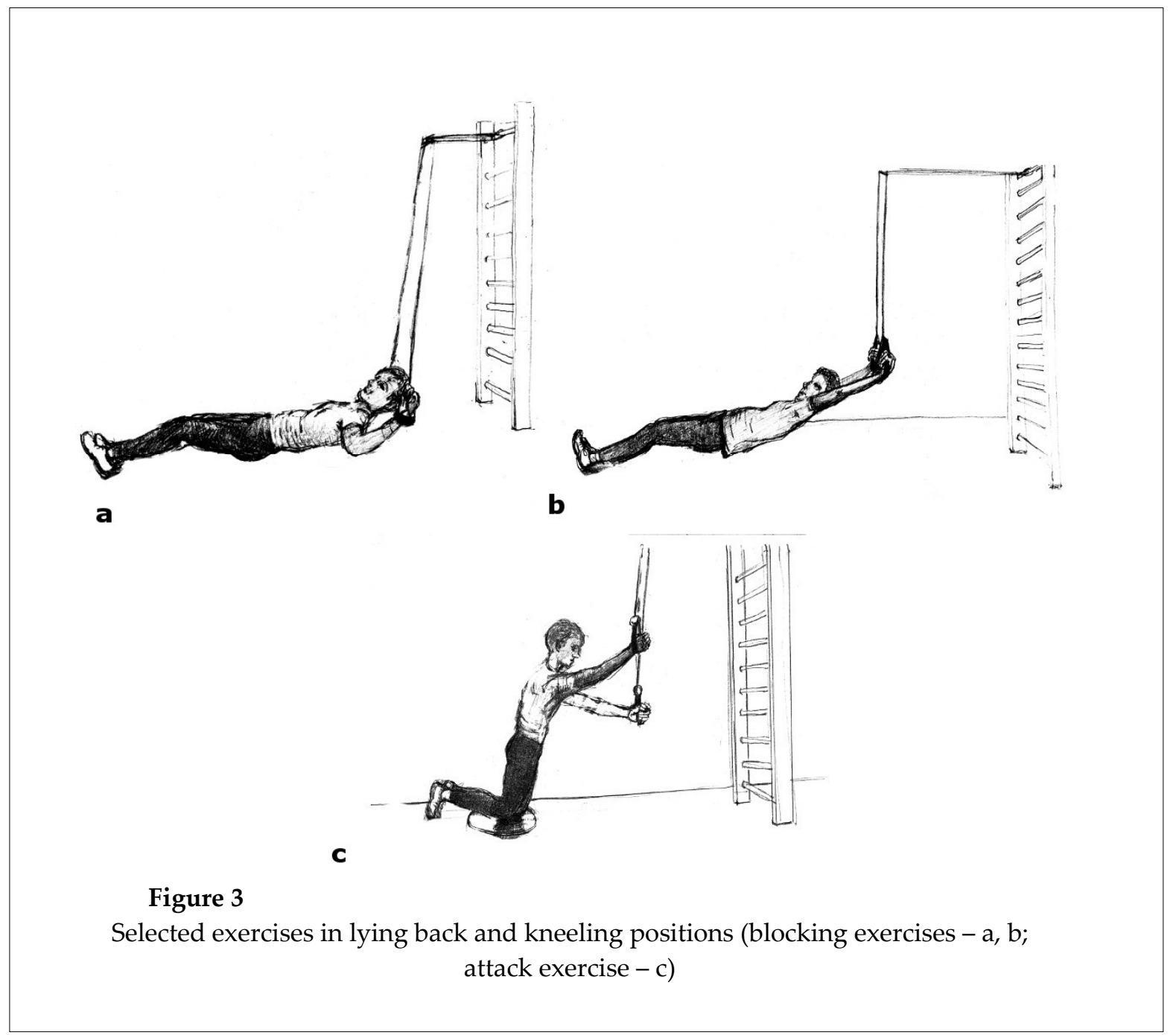

\begin{tabular}{|c|c|c|c|c|c|c|}
\hline & & & & \multicolumn{3}{|c|}{ Table 1} \\
\hline \multirow{2}{*}{\multicolumn{2}{|c|}{ Dependent variables }} & \multicolumn{3}{|c|}{ mean rang } & \multicolumn{2}{|c|}{ Friedman Test } \\
\hline & & First & Second & Third & $\mathrm{Ch}^{2}$ & $p$ \\
\hline \multirow{13}{*}{ 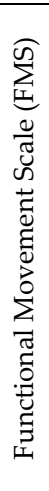 } & Deep squat & 1.83 & 1.83 & 2.33 & 10.0 & $<0.01^{*}$ \\
\hline & Hurdle step $-\mathrm{R}$ & - & - & - & - & - \\
\hline & Hurdle step - L & - & - & - & - & - \\
\hline & in-line lunge $-\mathrm{R}$ & 1.93 & 1.93 & 2.13 & 4.0 & 0.13 \\
\hline & In-line lunge - L & 1.96 & 1.96 & 2.06 & 2.0 & 0.37 \\
\hline & Shoulder mobility - L & 1.96 & 1.96 & 2.06 & 2.0 & 0.37 \\
\hline & Shoulder mobility - R & 1.96 & 1.96 & 2.06 & 2.0 & 0.37 \\
\hline & Active leg raise $-\mathrm{R}$ & 1.96 & 1.96 & 2.06 & 2.0 & 0.37 \\
\hline & Active leg raise $-\mathrm{L}$ & - & - & - & - & - \\
\hline & Push-up & 1.86 & 1.86 & 2.26 & 8.0 & $0.01^{*}$ \\
\hline & Rotatory stability $-\mathrm{R}$ & 1.63 & 1.63 & 2.73 & 22.0 & $<0.001^{*}$ \\
\hline & Rotatory stability - L & 1.70 & 1.70 & 2.60 & 18.0 & $<0.001^{*}$ \\
\hline & FMS - score & 1.60 & 1.60 & 2.80 & 24.0 & $<0.001^{*}$ \\
\hline \multicolumn{7}{|c|}{$\begin{array}{c}\text { First - initial FMS testing; Second - second FMS testing after two months; } \\
\text { Third - FMS testing after stabilisation training (two months after second FMS testing); } \\
\text { "-" the same results at each stage; } R \text { - right side; } L \text { - left side }\end{array}$} \\
\hline
\end{tabular}




\begin{tabular}{|c|c|c|c|c|c|c|c|c|c|c|c|c|c|}
\hline \multicolumn{14}{|c|}{ Table 2} \\
\hline & DS & HSR & HSL & LLR & LLL & SML & SMR & LRR & LRL & $\mathrm{PU}$ & RSR & RSL & FMS \\
\hline 1 & $2 / 2 / 2$ & $3 / 3 / 3$ & $3 / 3 / 3$ & $2 / 2 / 2$ & $2 / 2 / 2$ & $3 / 3 / 3$ & $3 / 3 / 3$ & $3 / 3 / 3$ & $3 / 3 / 3$ & $1 / 1 / 2$ & $1 / 1 / 2$ & $1 / 1 / 2$ & $16 / 16 / 17$ \\
\hline 2 & $2 / 2 / 2$ & $3 / 3 / 3$ & $3 / 3 / 3$ & $2 / 2 / 2$ & $2 / 2 / 2$ & $2 / 2 / 3$ & $3 / 3 / 3$ & $2 / 2 / 2$ & $2 / 2 / 2$ & $3 / 3 / 3$ & $1 / 1 / 2$ & $1 / 1 / 2$ & 14/14/17 \\
\hline 3 & $3 / 3 / 3$ & $3 / 3 / 3$ & $3 / 3 / 3$ & $2 / 2 / 2$ & $2 / 2 / 2$ & $3 / 3 / 3$ & $3 / 3 / 3$ & $3 / 3 / 3$ & $3 / 3 / 3$ & $3 / 3 / 3$ & $2 / 2 / 3$ & $2 / 2 / 3$ & $18 / 18 / 20$ \\
\hline 4 & $2 / 2 / 2$ & $2 / 2 / 2$ & $2 / 2 / 2$ & $2 / 2 / 2$ & $2 / 2 / 2$ & $2 / 2 / 2$ & $2 / 2 / 2$ & $2 / 2 / 2$ & $2 / 2 / 2$ & $2 / 2 / 2$ & $1 / 1 / 2$ & $1 / 1 / 2$ & $12 / 12 / 14$ \\
\hline 5 & $3 / 3 / 3$ & $3 / 3 / 3$ & $3 / 3 / 3$ & $2 / 2 / 2$ & $2 / 2 / 2$ & $3 / 3 / 3$ & $3 / 3 / 3$ & $3 / 3 / 3$ & $3 / 3 / 3$ & $2 / 2 / 3$ & $2 / 2 / 2$ & $2 / 2 / 2$ & 18/18/19 \\
\hline 6 & $2 / 2 / 3$ & $3 / 3 / 3$ & $3 / 3 / 3$ & $3 / 3 / 3$ & $3 / 3 / 3$ & $3 / 3 / 3$ & $3 / 3 / 3$ & $3 / 3 / 3$ & $3 / 3 / 3$ & $2 / 2 / 2$ & $3 / 3 / 3$ & $3 / 3 / 3$ & $19 / 19 / 20$ \\
\hline 7 & $2 / 2 / 2$ & $3 / 3 / 3$ & $3 / 3 / 3$ & $2 / 2 / 2$ & $2 / 2 / 2$ & $3 / 3 / 3$ & $3 / 3 / 3$ & $3 / 3 / 3$ & $3 / 3 / 3$ & $2 / 2 / 2$ & $1 / 1 / 2$ & $1 / 1 / 2$ & $16 / 16 / 17$ \\
\hline 8 & $3 / 3 / 3$ & $3 / 3 / 3$ & $3 / 3 / 3$ & $3 / 3 / 3$ & $3 / 3 / 3$ & $3 / 3 / 3$ & $3 / 3 / 3$ & $3 / 3 / 3$ & $3 / 3 / 3$ & $2 / 2 / 3$ & $2 / 2 / 3$ & $2 / 2 / 2$ & $19 / 19 / 20$ \\
\hline 9 & $2 / 2 / 3$ & $3 / 3 / 3$ & $3 / 3 / 3$ & $2 / 2 / 2$ & $2 / 2 / 2$ & $3 / 3 / 3$ & $2 / 2 / 3$ & $3 / 3 / 3$ & $3 / 3 / 3$ & $2 / 2 / 2$ & $2 / 2 / 2$ & $2 / 2 / 2$ & $17 / 17 / 18$ \\
\hline 10 & $1 / 1 / 2$ & $3 / 3 / 3$ & $3 / 3 / 3$ & $2 / 2 / 2$ & $2 / 2 / 2$ & $2 / 2 / 2$ & $3 / 3 / 3$ & $3 / 3 / 3$ & $3 / 3 / 3$ & $3 / 3 / 3$ & $2 / 2 / 2$ & $2 / 2 / 2$ & 17/17/17 \\
\hline 11 & $2 / 2 / 3$ & $3 / 3 / 3$ & $3 / 3 / 3$ & $2 / 2 / 2$ & $2 / 2 / 2$ & $3 / 3 / 3$ & $3 / 3 / 3$ & $2 / 2 / 3$ & $3 / 3 / 3$ & $1 / 1 / 2$ & $1 / 1 / 2$ & $1 / 1 / 2$ & 15/15/18 \\
\hline 12 & $2 / 2 / 3$ & $3 / 3 / 3$ & $3 / 3 / 3$ & $2 / 2 / 2$ & $2 / 2 / 2$ & $3 / 3 / 3$ & $3 / 3 / 3$ & $3 / 3 / 3$ & $3 / 3 / 3$ & $2 / 2 / 2$ & $2 / 2 / 3$ & $2 / 2 / 2$ & 18/18/18 \\
\hline 13 & $3 / 3 / 3$ & $3 / 3 / 3$ & $3 / 3 / 3$ & $2 / 2 / 3$ & $3 / 3 / 3$ & $3 / 3 / 3$ & $3 / 3 / 3$ & $3 / 3 / 3$ & $3 / 3 / 3$ & $2 / 2 / 2$ & $1 / 1 / 2$ & $1 / 1 / 2$ & 16/16/19 \\
\hline 14 & $2 / 2 / 2$ & $2 / 2 / 2$ & $2 / 2 / 2$ & $1 / 1 / 2$ & $1 / 1 / 2$ & $2 / 2 / 2$ & $3 / 3 / 3$ & $2 / 2 / 2$ & $2 / 2 / 2$ & $3 / 3 / 3$ & $1 / 1 / 2$ & $1 / 1 / 2$ & $11 / 11 / 17$ \\
\hline 15 & $3 / 3 / 3$ & $3 / 3 / 3$ & $3 / 3 / 3$ & $2 / 2 / 2$ & $2 / 2 / 2$ & $3 / 3 / 3$ & $3 / 3 / 3$ & $3 / 3 / 3$ & $3 / 3 / 3$ & $3 / 3 / 3$ & $2 / 2 / 3$ & $2 / 2 / 3$ & $18 / 18 / 20$ \\
\hline \multicolumn{14}{|c|}{$\begin{array}{l}\text { initial score/score after two months/score after completing } 8 \text { week stabilisation training; DS - } \\
\text { deep squat; HSR - hurdle step (right side); HSL - hurdle step (left side); LLR - in-line lunge } \\
\text { (right side); LLL - in-line lunge (left side); SMR - shoulder mobility (right side); SML - } \\
\text { shoulder mobility (left side); LRR - active straight leg raise (right side); LRL - active straight } \\
\text { leg raise (left side); PU - trunk stability push-up; RSR - rotary stability (right side); RSL - } \\
\text { rotary stability (left side) }\end{array}$} \\
\hline
\end{tabular}

\section{Discussion}

The aim of this study was to evaluate the effect of an eight-week stabilisation workout on FMS test results in adolescent volleyball players. To our knowledge, this was the first study where an attempt to assess such a relationship was made. The results of the research show that the applied stabilisation training significantly improved subjects' scores in the following tests: a deep squat, a trunk stability push-up, a rotator stability test on both sides of the body, as well as the total FMS test score.

In the studied population, the average FMS test score before stabilisation training was 16.3 , i.e. slightly higher than the results obtained by the adult population (Schneiders et al., 2011) as the average value of adult males equaled 15.8 points. Examining young male athletes between 8 and 14 years of age, the mean value of the FMS test was 15.2 points (Paszkewicz et al., 2013), which is also a lower value than the one obtained in this study. Perhaps better FMS test results in the studied population, as compared to the results of Paszkiewicz et al. (2013), stem from the fact that subjects from the present study were at the same age, while in Paszkewicz et al.'s (2013) study, subjects' age varied from 8 to 14 years. In turn, better FMS test results obtained in the present study compared to the adult population may be due to a higher level of physical activity. In the research of Schneiders et al. (2011), the subjects were physically active males between 18 and 40 years of age, whereas in the present study the subjects were youth competitive athletes practicing volleyball ( $4.5 \mathrm{~h}$ per week). In other studies, active males between 18 and 25 years old recorded a FMS result of 16.7 (Letafatkar et al., 2014) and professional soccer players scored up to 16.9 (Kiesel et al., 2007). These results are similar to those found in the present study.

In the first and the second measurement, $20 \%$ of young male subjects achieved a total score of no more than 14 points on the FMS test. Analyzing results of other studies, although in 
adult populations, Schneiders et al. (2011) observed that $31 \%$ of adult males had a score of 14 or less on the FMS test, while Letafatkar et al. (2014) found that $27 \%$ of the subjects (mean age: 22.6 years) obtained a score of no more than 14 points. However, among professional soccer players, the score of 14 points or less was recorded by in $22 \%$ of the subjects (Kiesel et al., 2007). Thus, despite a much younger population in the present study, a similar percentage of subjects with a number of $\leq 14$ points on the FMS test was achieved.

Tests assessing the risk of injury suggest that athletes who have received 14 or fewer points on the FMS test are more likely to sustain an injury (specificity of 0.91 and sensitivity of 0.54 ). The odds of sustaining an injury were 17.7 times higher in those with an FMS score $\leq 14$ compared with those with a score of $>14$ points (Kiesel et al., 2007). Thus, the research of Kiesel et al. (2007) indicates that such a low FMS test result is associated with a higher risk to injury. Furthermore, the study of Letafatkar et al. (2014) also suggests that a better FMS test result may still indicate greater likelihood of injury. That study showed that subjects who scored less than 17 on the FMS were 4.7 times more likely to sustain an injury of the lower extremity. In the present study, $47 \%$ of the subjects obtained an FMS score below 17 points during first and second testing. From this point of view, it is necessary to establish appropriate training which would improve FMS scores in athletes.

Stabilization training applied to the population of boys at the age of 14 years resulted in a significant improvement of their performance during FMS testing as only one person obtained the result of below 14 points, and only $6.7 \%$ of the subjects had the result of below 17 points. On this basis, it can be assumed that eight-week stabilization training can affect the results of the FMS tests and provide some form of protection against possible injuries. In another work, Wright et al. (2014) applied fundamental movement training to adolescents over a period of four weeks, and obtained contradictory results, which suggests that short-term interventions might affect specific isolated components of fitness, but not FMS performance. However, sling exercises using the NEURAC method promote activation of nerve roots, smooth coordination between the trunk and the hip joint and reduce an abnormal flexion-relaxation phenomenon (Kirkesola, 2009), which also has a positive effect on muscle responses in a closed kinematic chain improving postural adjustment ability. It seems that to improve FMS performance more than four weeks of NEURAC training is needed.

Further assessment of the impact of various forms of exercise on the FMS test results should be the subject of future studies. For this research, the design applied allowed the experimental group to be the control group for itself after being monitored for four months. Risks associated with the procedure may be related with the transfer effect; however, in the present study there was no visible difference between the first and the second series of measurements. Another limitation concerns the studied population. In the present study only a small sample was investigated, and this group underwent specific training related to the sport practiced. Thus, the results as well as the training program applied can refer only to groups that practice volleyball. Consideration should be also given to the gender of the subjects. In this paper, we studied only young males and applying the same training protocol in females may have brought different results.

In conclusion, 8 week stabilization training designed specifically for volleyball players affects the FMS test score in young males practicing volleyball, mainly the deep squat, push-up and rotatory stability test results.

\section{Practical implications}

The applied stabilisation exercises based on the NEURAC method positively influenced the FMS test performance. Conducted training resulted in more than $90 \%$ of the subjects to have a total FMS test score $\geq 17$, which may be important for the prevention of injuries.

The results of the study suggest that exercises in a closed kinematic chain on an unstable ground (as in the NEURAC sling exercises method) should be taken into account during normal sport training. 


\section{References}

Chorba RS, Chorba DJ, Bouillon LE, Overmyer CA, Landis JA. Use of a functional movement screening tool to determine injury risk in female collegiate athletes. N Am J Sports Phys Ther, 2010; 5(2): 47-54

Cook G, Burton L, Hoogenboom B. Pre-participation screening: the use of fundamental movements as an assessment of function - part 1. N Am J Sports Phys Ther, 2006a; 1(2): 62-72

Cook G, Burton L, Hoogenboom B. Pre-participation screening: the use of fundamental movements as an assessment of function - part 2. N Am J Sports Phys Ther, 2006b; 1(3): 132-139

Kiesel K, Plisky P, Butler R. Functional movement test scores improve following a standardized off-season intervention program in professional football players. Scand J Med Sci Sports, 2011; 21(2): 287-292

Kiesel K, Plisky P, Kersey P. Functional Movement Test Score as a Predictor of Time- loss during a Professional Football Team's Pre- season. Paper presented at: American College of Sports Medicine Annual Conference 2008; Indianapolis, IN

Kiesel K, Plisky PJ, Voight ML. Can Serious Injury in Professional Football be Predicted by a Preseason Functional Movement Screen? N Am J Sports Phys Ther, 2007; 2(3): 147-158

Kirkesola G. Neurac-a new treatment method for long-term musculoskeletal pain. Fysioterapeuten, 2009; 76: $16-25$

Kirkesola G. Sling exercise therapy (S-E-T): a total concept for exercise and active treatment of musculoskeletal disorders. J Korean Acad Orthop Man Ther, 2001; 7: 87-106

Letafatkar A, Hadadnezhad M, Shojaedin S, Mohamadi E. Relationship between functional movement screening score and history of injury. Int J Sports Phys Ther, 2014; 9(1): 21-27

Narducci E, Waltz A, Gorski K, Leppla L, Donaldson M. The clinical utility of functional performance tests within one-year post-acl reconstruction: a systematic review. Int J Sports Phys Ther, 2011; 6(4): 333-342

Paszkewicz JR, McCarty CW, Van Lunen BL. Comparison of functional and static evaluation tools among adolescent athletes. J Strength Cond Res, 2013; 27(10): 2842-2850

Schneiders AG, Davidsson A, Hörman E, Sullivan SJ. Functional movement screen normative values in a young, active population. Int J Sports Phys Ther, 2011; 6(2): 75-82

Stanton R, Reaburn PR, Humphries B. The effect of short-term Swiss ball training on core stability and running economy. J Strength Cond Res, 2004; 18(3): 522-528

Wright MD, Portas MD, Evans VJ, Weston M. The effectiveness of four weeks of fundamental movement training on Functional Movement Screen ${ }^{\mathrm{TM}}$ and physiological performance in physically active children. J Strength Cond Res 2014; 6(28) [Epub ahead of print]

Zazulak BT, Hewett TE, Reeves NP, Goldberg B, Cholewicki J. Deficits in neuromuscular control of the trunk predict knee injury risk: a prospective biomechanical-epidemiologic study. Am J Sports Med, 2007a; 35(7): 1123-1130

Zazulak BT, Hewett TE, Reeves NP, Goldberg B, Cholewicki J. The effects of core proprioception on knee injury: a prospective biomechanical-epidemiological study. Am J Sports Med, 2007b; 35(3): 368-373

\section{Corresponding author:}

\section{Linek Pawel}

Department of Kinesitherapy and Special Methods in Physiotherapy

The Jerzy Kukuczka Academy of Physical Education, 40-065, Mikolowska 72B, Poland

Phone: +48 661768601

E-mail: linek.fizjoterapia@vp.pl 